\title{
Bullying e cyberbullying entre adolescentes: protótipo de game para adolescentes no
}

\section{ambiente escolar}

\author{
Bullying and cyberbullying among adolescents: prototype game for adolescents in the school \\ environment
}

Bullying y ciberacoso entre adolescentes: prototipo de juego para adolescentes en el entorno escolar

Recebido: 13/01/2021 | Revisado: 15/01/2021 | Aceito: 21/01/2021 | Publicado: 25/01/2021

\author{
Claudinalle Farias Queiroz de Souza \\ ORCID: https://orcid.org/0000-0003-1541-3089 \\ Universidade de Pernambuco, Brasil \\ E-mail: claudinalle.souza@upe.br \\ Maria Vanessa Nascimento dos Anjos \\ ORCID: https://orcid.org/0000-0001-5411-4431 \\ Universidade de Pernambuco, Brasil \\ E-mail: vanessanascanjos@gmail.com \\ Maria Aparecida Beserra \\ ORCID: https://orcid.org/0000-0002-5315-5589 \\ Universidade de Pernambuco, Brasil \\ E-mail: aparecida.beserra@upe.br \\ Waldemar Brandão Neto \\ ORCID: https://orcid.org/ 0000-0003-4786-9961 \\ Universidade de Pernambuco, Brasil \\ E-mail: waldemar.neto@upe.br \\ Ana Carolina Pereira Gomes \\ ORCID: https://orcid.org/0000-0002-8400-6287 \\ Universidade de Pernambuco, Brasil \\ E-mail: anacarolinagomes07@gmail.com
}

\begin{abstract}
Resumo
O objetivo deste estudo é elaborar um protótipo de um game que contribua com a educação em saúde sobre o Bullying e Cyberbullying entre adolescentes no ambiente escolar. Pesquisa de produção tecnológica baseada no design thinking, desenvolvida em quatro etapas: 1 . Análise dos dados do questionário bullying; 2 . Revisão sistemática de literatura; 3. Pesquisa de aplicativos ou games disponíveis com temática similar na Google Play Store e App Store; 4. Elaboração de um protótipo de um game no estilo simulação. 99 estudantes responderam ao questionário. Na revisão sistemática, 13 artigos foram incluídos. Na busca dos aplicativos/games, 42 aplicativos foram analisados. O game de simulação é chamado B.S.I.: Bullying Scene Investigation. A temática é de investigação, na qual o participante busca na escola pistas de possíveis situações de bullying e cyberbullying e notifica à direção. O game pode produzir maior conscientização sobre o contexto de bullying, uma vez que possibilita promover o protagonismo do estudante no espaço escolar, o engajamento no combate à violência, a motivação para ajudar as vítimas de bullying e a colaboração com o professor e gestão escolar nas estratégias de enfrentamento apropriadas à realidade.
\end{abstract}

Palavras-chave: Bullying; Cyberbullying; Saúde do adolescente; Jogos recreativos; Serviços de enfermagem escolar.

\begin{abstract}
The aim of this study is to develop a prototype of a game that contributes to health education about Bullying and Cyberbullying among adolescents in the school environment. Technological production research based on design thinking, developed in four stages: 1. Analysis of the data of the bullying questionnaire; 2. Systematic literature review; 3. Search for apps or games available with similar theme in the Google Play Store and App Store; 4. Develop a prototype of a game in simulation style. 99 students answered the questionnaire. In the systematic review, 13 articles were included. In the search of the applications/games, 42 applications were analyzed. The simulation game is called B.S.I.: Bullying Scene Investigation. The theme is investigation, in which the participant searches in the school for clues of possible situations of bullying and cyberbullying and notifies the direction. The game can produce greater awareness about the context of bullying, since it allows promoting the student's protagonism in the school space, the engagement in the fight against violence, the motivation to help victims of bullying and collaboration with the teacher and school management in the strategies of appropriate coping.
\end{abstract}

Keywords: Bullying; Cyberbullying; Adolescent health; Recreational games; School nursing services. 


\section{Resumen}

El objetivo de este estudio es crear un juego que contribuya a la educación sanitaria sobre Bullying y Cyberbullying entre los adolescentes en el entorno escolar. Investigación de producción tecnológica basada design thinking, desarrollada en cuatro etapas: 1 . Análisis de los datos del cuestionario bullying; 2. Revisión sistemática de la literatura; 3. Buscar aplicaciones o juegos disponibles con un tema similar en Google Play Store y App Store; 4. Crear un game en estilo de simulación. 99 estudiantes respondieron al cuestionario. En el examen sistemático, se incluyeron 13 artículos. En la búsqueda de las aplicaciones/juegos, se analizaron 42 aplicaciones. El game de simulación se llama B.S.I.: Bullying Scene Investigation. El tema es la investigación en la que el participante busca en la escuela pistas de posibles situaciones de bullying y cyberbullying y notifica la dirección. El game puede producir una mayor conciencia sobre el contexto del bullying, ya que permite promover el protagonismo del alumno en el espacio escolar, el compromiso en la lucha contra la violencia, la motivación para ayudar a las víctimas del bullying y la colaboración con el profesor y la dirección escolar en las estrategias de colaborar adecuadamente.

Palabras clave: Bullying; Cyberbullying; Salud de los adolescentes; Juegos recreativos; Servicios de enfermería escolar.

\section{Introdução}

As diretrizes nacionais para a atenção integral à saúde de adolescentes e de jovens foram construídas com o intuito de estimular estratégias intersetoriais que visem à modificação do cenário de vulnerabilidade desse grupo populacional. Um dos temas estruturantes dessa diretriz é a cultura de paz, que aborda os diferentes tipos de violência aos quais esta população está exposta, assim como sua participação nesse ciclo seja como autor, vítima ou testemunha (Diretrizes Nacionais para a Atenção Integral à Saúde de Adolescentes e Jovens na Promoção, Proteção e Recuperação da Saúde, 2010).

O bullying é caracterizado por uma ação de violência desigual que busca incomodar e humilhar outra pessoa seja de forma física ou verbal, e o cyberbullying configura-se por atos de agressão através dos meios eletrônicos (correios eletrônicos, sites, redes sociais, mensagens e/ou telefonemas) (Wendt \& Lisboa, 2013).

Ambos são um grave problema, pois impactam as emoções, o comportamento e o bem-estar psicológico não apenas das vítimas, mas dos próprios agressores e espectadores (Panumaporn, Hongsanguansri, Atsariyasing, \& Kiatrungrit, 2020).

É notável a necessidade de elaboração de programas que visem à prevenção da violência e a promoção da saúde da escola. Umas das estratégias de educação em evidência é o uso da gamificação como forma de aproximar os adolescentes aos assuntos abordados e obter um maior engajamento, contribuindo para a promoção da saúde. A gamificação utiliza uma série de elementos de jogo (dinâmica, mecânica e estética) para chamar a atenção do jogador para um questionamento específico, e esta pode ser uma ótima ferramenta para combater o bullying (Ortiz-Colón, Jordán, \& AgredaI, 2018).

Diante deste contexto, o objetivo desta pesquisa foi elaborar o protótipo de um game que contribua com a educação em saúde sobre o Bullying e Cyberbullying entre adolescentes no ambiente escolar.

\section{Metodologia}

Trata-se de um estudo de produção tecnológica, com abordagem quantitativa para o desenvolvimento de um protótipo de game educacional na prevenção do bullying e cyberbullying. Neste tipo de estudo, o foco volta-se para a construção e validação de ferramentas e métodos aplicáveis a melhorias na realidade de saúde e cuidado das pessoas (Rosa, Locks, \& Costa, 2019). Sua realização ocorreu entre os meses de março a novembro de 2019, na Faculdade de Enfermagem Nossa Senhora das Graças da Universidade de Pernambuco - FENSG/UPE e na Escola Pública Estadual José Maria, ambas localizadas no bairro de Santo Amaro, no município de Recife, em Pernambuco. A escola foi selecionada de forma aleatória através de sorteio das instituições de ensino públicas localizadas no referido bairro. O bairro foi selecionado devido à presença de alto índice de violência no contexto escolar (Beserra et al., 2019). 


\section{Fases do desenvolvimento}

O processo de produção tecnológica foi baseado na estratégia do design thinking (Vianna et al., 2012) composta das fases de imersão (análise dos dados do questionário bullying e revisão sistemática), ideação (análise dos games) e elaboração de um protótipo.

A fase de imersão consistiu na análise de dados coletados presencialmente com os adolescentes através do questionário adaptado de Dan Olwes (1996) de identificação de Bullying e Cyberbullying, (Gonçalves et al., 2016) esta etapa foi realizada em fase anterior da pesquisa. Uma amostra por conveniência de 99 adolescentes de ambos os sexos e matriculados nos anos finais do ensino fundamental completou as respostas do questionário. Estes dados foram utilizados a fim de trazer embasamento teórico sobre o perfil dos adolescentes a que se pretendia atender com o game. As questões analisadas corresponderam aos dados de idade, sexo, ano escolar e se já havia presenciado uma situação de violência escolar.

Continuando nesta fase, foi realizada uma revisão sistemática fundamentada nas recomendações da metodologia Principais Itens para Relatar Revisões sistemáticas e Meta-análises - PRISMA (Moher, Liberati \&Tetzlaff, 2015). Os termos de busca foram selecionados na base de dados dos Descritores de Ciências da Saúde (DeCS) e seus similares no Medical Subjects Headings (MeSH), nos idiomas português e inglês, sendo eles: adolescente, bullying, jogos experimentais, jogos recreativos e teoria do jogo. Para associação dos descritores, utilizou-se o operador booleano AND.

As bases de dados selecionadas foram: Medical Literature Analysis and Retrieval System Online (MEDLINE), Literatura Latino-Americana e do Caribe em Ciências da Saúde (LILACS), e Banco de Dados em Enfermagem (BDENF). As buscas ocorreram entre junho e julho de 2019. Foram incluídos artigos científicos disponíveis na íntegra com acesso aberto, publicados entre 2014 a 2018, e nos idiomas português e inglês. Os critérios de exclusão foram artigos em duplicidade nas bases de dados e que não apresentassem similaridade com o objeto de estudo por meio da leitura de título e resumo.

A ideação foi composta por uma pesquisa de outros aplicativos ou games disponíveis com temática similar na Google Play Store e App Store, desenvolvidos nos sistemas operacionais (SO) Android e iOS. Foram classificados quanto ao tipo (aplicativo ou game), seu objetivo, categoria, faixa etária do público alvo e a disponibilidade (gratuito ou pago).

$\mathrm{Na}$ fase de elaboração de um protótipo, relativamente aos conceitos, cenários e personagens incluídos no protótipo de game, fundamentou-se em dois importantes eixos teóricos, a saber: o Programa Saúde na Escola (PSE) e a Lei brasileira Antibullying de ${ }^{\circ}$ 13.185/2015 que institui o Programa de Combate à Intimidação Sistemática nas escolas do país. O tipo de game produzido foi o de simulação. O software escolhido foi o Scratch, que é um projeto desenvolvido no Massachusetts Institute of Technology - MIT que se constitui de uma construção de histórias, animações, jogos e simuladores a partir de comandos construídos em formato de blocos (Sápiras, Vecchia, \& Maltempi, 2015).

\section{Análise dos dados}

Os dados foram tabulados no Microsoft Excel a partir das respostas dos participante,s e então analisados por meio de estatística descritiva em frequências absolutas, relativas e médias.

\section{Considerações éticas}

Esta pesquisa é parte integrante de uma pesquisa principal e foi aprovada pelo Comitê de Ética em Pesquisa da HUOC/PROCAPE, segundo a Resolução 466/12 do Conselho Nacional de Saúde, sob parecer nº 2.305.054.

\section{Resultados e Discussão}

Responderam aos questionários 99 estudantes, a maioria era do sexo feminino (56,5\%). A faixa etária predominante foi de 12 a 13 anos $(60,6 \%)$, o que corresponde a idade escolar do $7^{\circ}$ ano do ensino fundamental (Tabela 1 ). 
Tabela 1 - Relação sexo, idade e número de estudantes. Recife, PE, 2019.

\begin{tabular}{|c|c|c|c|c|c|c|}
\hline Idade (anos) & Feminino (n) & Feminino \% & Masculino (n) & Masculino \% & $\begin{array}{c}\text { Número de estudantes } \\
\text { n }\end{array}$ & $\begin{array}{c}\text { Número de } \\
\text { estudantes } \\
\%\end{array}$ \\
\hline 11 & 5 & 8,93 & 3 & 6,98 & 8 & 8,08 \\
\hline 12 & 21 & 37,50 & 13 & 30,23 & 34 & 34,34 \\
\hline 13 & 17 & 30,36 & 9 & 20,93 & 26 & 26,26 \\
\hline 14 & 8 & 14,29 & 7 & 16,28 & 15 & 15,15 \\
\hline 15 & 5 & 8,93 & 7 & 16,28 & 12 & 12,12 \\
\hline 16 & 0 & 0,00 & 1 & 2,33 & 1 & 1,01 \\
\hline 17 & 0 & 0,00 & 1 & 2,33 & 1 & 1,01 \\
\hline 19 & 0 & 0,00 & 2 & 4,65 & 2 & 2,02 \\
\hline Total & 56 & 100 & 43 & 100 & 99 & 100 \\
\hline
\end{tabular}

Fonte: Dados da pesquisa (2019).

Aproximadamente 29,6\% dos estudantes do sexo feminino com idades de 11 a 15 anos relataram terem sido vítimas de bullying. Para os estudantes do sexo masculino na mesma faixa etária, a porcentagem de vítimas foi de 52,9\%. Há estudos que apontam as diferentes formas de violência sofrida pelos meninos e meninas - enquanto elas sofrem mais com o bullying indireto (fofocas, difamações), os meninos são acometidos pelo bullying direto (violência física ou ameaças) (Silva, Dascanio, \& Valle, 2016).

O questionário de indicadores de bullying apontou que $81,8 \%$ dos escolares já haviam presenciado uma situação de violência no ambiente escolar e que os agentes sempre eram os próprios alunos. Em torno de 52,0\% dos entrevistados afirmam terem sido vítimas de mentiras e boatos, sendo que 5,0\% deles alegaram sofrer com essa violência diariamente. Além disso, os apelidos pejorativos também dificultam a convivência escolar dos alunos, visto que, ao todo, 18 alunos foram menosprezados pelos seus colegas por causa de sua aparência e/ou deficiência física.

No estudo de Nomelini et al (2020), foi observado um percentual de aproximadamente 53\% de vítimas do sexo masculino, maior na escola privada. Entre as agressões se destacaram os apelidos (48\%) e os insultos pelas características físicas $(48 \%)$, seguidos de dizer coisas negativas sobre a pessoas ou família (34\%) e a agressão física (12\%). O bullying afeta negativamente o clima escolar e o desenvolvimento de todos os envolvidos - agressor, vítima e toda a comunidade escolar.

O bullying verbal foi a forma de violência que mais vitimizou os adolescentes analisados, uma possível explicação para essa prevalência pode estar na ausência de políticas públicas claras de combate ao bullying (Silva et al., 2019). A vitimização do bullying tem sido associada a uma série de resultados adversos de saúde e sociais, incluindo problemas de saúde mental, porte de armas, abuso de substâncias, problemas acadêmicos e outras consequências adversas, algumas das quais podem persistir na idade adulta (Programa Saúde na Escola: Caderno do Gestor do PSE, 2015).

Como demonstram os dados, as situações de violência nos ambientes escolares estão se tornando algo recorrente. Internacionalmente, o Brasil está entre os dez países com maiores taxas de homicídios entre jovens de 15 a 19 anos. Como consequência da violência interpessoal, observam-se internações decorrentes de ferimentos físicos, piora na qualidade de vida, transtorno de estresse pós-traumático, depressão, ideação suicida e mortes (Silva et al., 2019).

Nos indicadores de cyberbullying levantados neste estudo, 56,3\% dos estudantes gastam de 15 a 19 horas ou mais por semana acessando à internet. Atualmente, os casos de cyberbullying crescem exponencialmente devido à quantidade exorbitante de tempo que os adolescentes gastam nas mídias sociais e afins. Partindo desse pressuposto, os adolescentes foram 
questionados se já haviam sido insultados através do correio eletrônico, e $22 \%$ deles afirmaram terem sofrido essa violência pelo menos uma vez.

Outro indicador analisado foi a exclusão em grupos ou jogos online. Nessa análise, 14,1\% estudantes relataram terem sido excluídos das interações online até três vezes. $\mathrm{O}$ uso de imagens sem autorização tornou-se um indicador de cyberbullying recorrente no ambiente escolar, visto que $14,1 \%$ estudantes relataram terem suas fotos e vídeos divulgados sem que eles tivessem conhecimento.

O bullying e o cyberbullying no âmbito escolar são exemplos de violências recorrentes e persistentes que causam problemas de saúde entre os adolescentes (Programa Saúde na Escola: Caderno do Gestor do PSE, 2015). São comportamentos agressivos prejudiciais nos quais algumas crianças e adolescentes infligem intencionalmente danos frequentes e de longo prazo aos colegas que se tornam vítimas.

\section{Revisão sistemática}

A revisão sistemática foi realizada para o embasamento teórico do game. Foram identificados 654 artigos, sendo 636 da base de dados Medline (97,24\%), 17 artigos na LILACS (2,59\%) e dois na BDENF (0,3\%). Destes, 624 artigos foram excluídos, seis por duplicação, 618 por não abordarem o tema. Para a fase da elegibilidade, foram escolhidos 30 artigos para análise de título e resumo. Com isso, 17 artigos foram eliminados pois o objetivo do estudo não condizia com o da revisão. Portanto,13 artigos se encaixaram nos critérios de inclusão estabelecidos (Figura 1).

Figura 1. Fluxograma da revisão sistemática segundo as recomendações PRISMA.

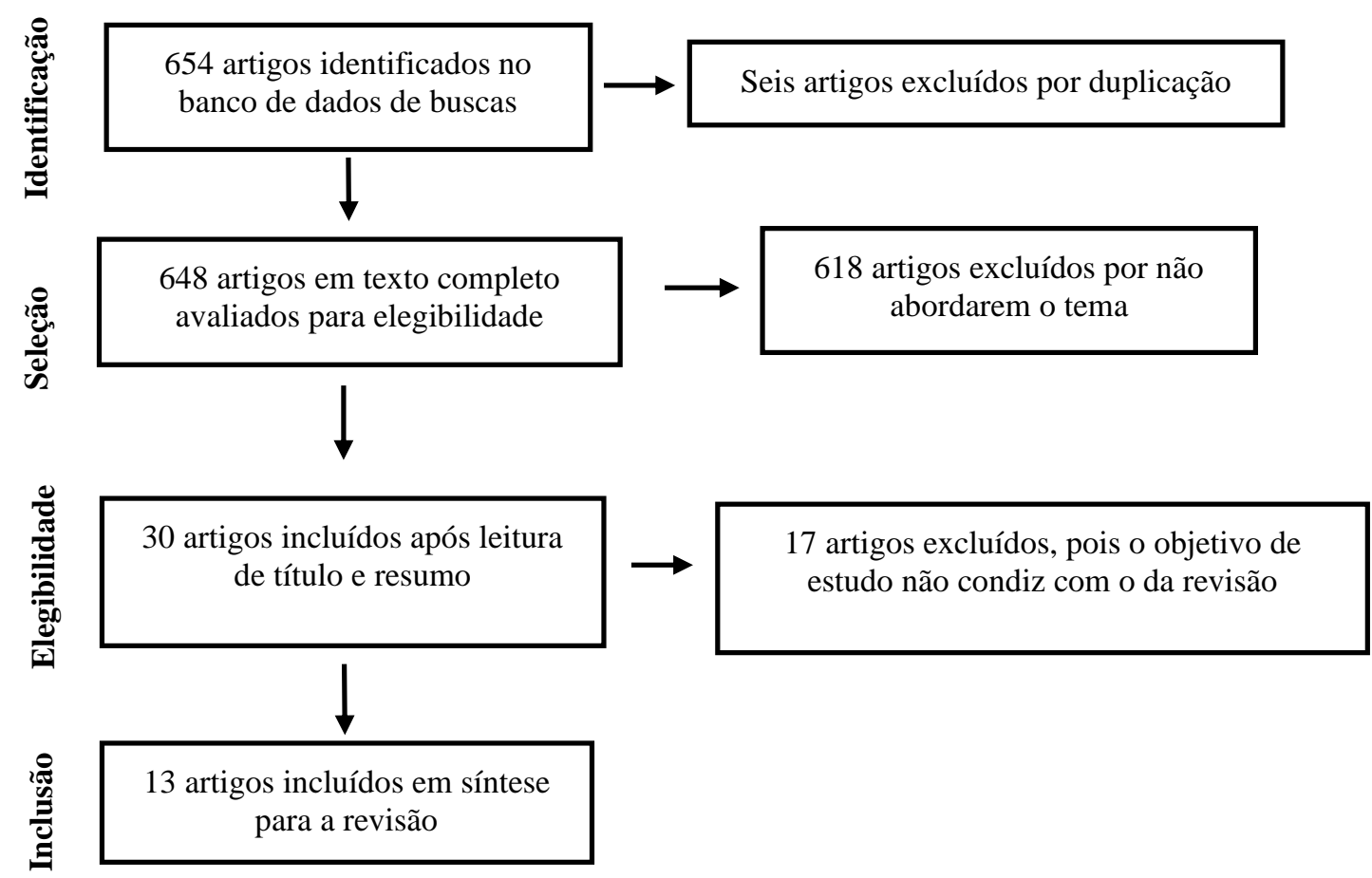

Fonte: Autores.

Dos 13 artigos selecionados para o estudo, 84,6\% foram publicados na língua inglesa e os demais na língua portuguesa. O público-alvo de maior prevalência foi o de adolescentes. A validação dos games propostos nesses estudos foi 
realizada pelo público-alvo excetuando dois artigos que tiveram seus games validados por um grupo de especialistas (enfermeiros), como apresentado no Quadro 1.

Quadro 1 - Quadro descritivo com as principais características relacionadas aos artigos da revisão sistemática.

\begin{tabular}{|c|c|c|c|c|c|}
\hline Tema & Autor, Ano & Idade & População & $\begin{array}{l}\text { Amostra } \\
\text { (n) }\end{array}$ & Tipo de jogo \\
\hline $\begin{array}{l}\text { Relacionamentos e } \\
\text { sexualidade }\end{array}$ & Bayley, 2015 & $20-61$ & Pais & 180 & Simulação \\
\hline $\begin{array}{c}\text { Impedir a propagação de um } \\
\text { vírus }\end{array}$ & Derex, 2016 & $18-44$ & Estudantes & 144 & Combinação \\
\hline Jogo de ping pong & Gorsic, 2017 & $18-80$ & $\begin{array}{l}\text { Pessoas com lesões } \\
\text { neurológicas }\end{array}$ & 35 & Ação \\
\hline Alimentação saudável & Marchetti, 2015 & $14-18$ & Adolescentes & 83 & Ação \\
\hline $\begin{array}{l}\text { Administração de } \\
\text { medicamentos }\end{array}$ & Moreira, 2014 & $18-27$ & $\begin{array}{l}\text { Estudantes de } \\
\text { Enfermagem }\end{array}$ & 40 & Tabuleiro \\
\hline Pólio & Okitika, 2015 & $20-61$ & Leigos em saúde & 164 & Tabuleiro \\
\hline Sexualidade & Sousa, 2018 & $12-18$ & Enfermeiros & 16 & Cartas \\
\hline Drogas ilícitas & Stapinski, 2017 & $14-17$ & Estudantes & 133 & Simulação \\
\hline Bebida alcoólica & Jander, 2016 & $15-19$ & Estudantes & 281 & Ação \\
\hline Desafios de vida & Fiellin, 2016 & $11-14$ & $\begin{array}{l}\text { Estudantes e } \\
\text { adolescentes }\end{array}$ & 333 & Simulação \\
\hline Saúde Sexual e Reprodutiva & Jozkowski, 2015 & $18-35$ & Universitários & 141 & Simulação \\
\hline Vida Social & Vrijhof, 2016 & $9-17$ & Estudantes & 38 & Ação \\
\hline Diabetes tipo 1 & Joubert, 2016 & $11-18$ & $\begin{array}{c}\text { Pacientes com diabetes } \\
\text { tipo } 1\end{array}$ & 2.649 & Simulação \\
\hline
\end{tabular}

Fonte: Autores.

Com relação aos tipos dos games desenvolvidos, observou-se aquele visando estimular o diálogo entre pais e filhos What Should We Tell the Children? (Bayley \& Brown, 2015), um que permite manipular a realidade e construir fórmulas virtual (Derex \& Boyd, 2016), o com sistemas de pontos/recompensa - Competitive arm rehabilitation game / Gustavo's in Gnam Planet Game (Goršič, Cikajlo, Goljar, \& Novak, 2017)(Marchetti et al., 2015), de tabuleiro - Polio eradication (Okitika et al., 2015)(Moreira, Sabóia, Camacho, Daher, \& Teixeira, 2014) de cartas - "Contando bem que mal tem?" (Sousa et al., 2018), de simulação utilizando avatar em cenas baseadas na realidade - Pure Rush / "What happened?" (Stapinski et al., 2017)(Jander, Crutzen, Mercken, Candel, \& De Vries, 2016), de simulação para mudança de comportamento em espaços de convívio social - Playforward: Elm City Stories / Campus craft (Fiellin et al., 2016)(Jozkowski \& Ekbia, 2015), que é em equipe para aumentar a empatia - Prosocial Cyberball Game (Vrijhof et al., 2016) e para o tratamento de doenças crônicas/estimular o autocuidado - "L'Affaire Birman" (Joubert et al., 2016).

Foi possível também identificar os principais temas abordados nos games, como saúde sexual e reprodutiva, uso de drogas, alimentação saudável, uso de medicamentos, convívio social e família e autocuidado nas doenças crônicas comuns na infância, porém houve ausência do tema Bullying e Cyberbullying (Bayley \& Brown, 2015). Este resultado reforça a necessidade desta pesquisa visando apresentar uma oportunidade de discussão nas escolas sobre este tema, através de um 
ambiente lúdico. Vale ressaltar que, um ponto em comum entre os games foi a preocupação em fortalecer alguma competência nas crianças e adolescentes e mudar comportamentos, algo peculiar nesta fase do desenvolvimento humano.

É de conhecimento que o cyberbullying vem ganhando um espaço significativo nos dados de violência escolar, isso acontece porque o ambiente virtual favorece a desinibição e oferta a possibilidade de anonimato através de identidade alternativa (uso de perfis falsos) para assediar. Assim, em alguns casos quem sofre bullying pode ser um perpetrador de cyberbullying contra seu algoz de bullying face a face, principalmente quando essa "revanche" não seria possível acontecer, como entre sujeitos com diferença de padrões físicos. As formas mais prejudiciais e negativas para as vítimas são os usos não autorizados de meios visuais (fotos e vídeos) (Ferreira \& Deslandes, 2018).

Entre as psicopatologias sofridas pelas vítimas de cyberbullying estão: insônia, depressão, falta de apetite, baixo rendimento escolar e/ou baixa concentração. Dessa forma, os estudantes têm seu processo de aprendizagem prejudicado e tornam-se cada vez mais vulneráveis (Pham \& Adesman, 2015).

Nos artigos revisados, os autores utilizaram diversos temas e contextos para produzir seus games, visando sempre à educação em saúde do público a quem ele era destinado, os eixos centrais trabalhados em cada jogo serviram de base para estruturar o protótipo de game proposto neste estudo. A partir destes dados, compreende-se que a gamificação cria meios para colaborar com a aprendizagem e promover a intervenção na saúde (Okitika et al., 2015).

\section{Análise dos games}

A segunda fase do design thinking, a ideação, foi realizada pela busca dos aplicativos/games nas lojas virtuais Google Play Store e App Store, utilizando o descritor bullying, e foram encontrados 42 aplicativos. Dentre os achados, quanto à categoria, $33(78,5 \%)$ eram educacionais, nove $(21,4 \%)$ para saúde. $35(83,3 \%)$ proporcionavam download free e sete $(16,6 \%)$ eram pagos. Porém, apenas dois eram games, um disponível somente no idioma inglês e outro em português, estes foram os avaliados e descritos no Quadro 2.

Quadro 2. Aplicativos que ofertam game de combate ao bullying.

\begin{tabular}{|c|c|c|c|c|}
\hline Título & Objetivo & Classificação & Categoria & $\begin{array}{c}\text { Tipo de } \\
\text { game }\end{array}$ \\
\hline No Bullying & $\begin{array}{c}\text { Combater o Bullying nas instituições de ensino, } \\
\text { por meio de textos objetivos, vídeos curtos, } \\
\text { possibilidade de auditoria e canal de denúncias, } \\
\text { conectada com os tempos atuais. }\end{array}$ & $12+$ & Educacional & Quiz \\
\hline Take a stand together & $\begin{array}{c}\text { Combater as diferentes situações de bullying por } \\
\text { meio de vídeos interativos, textos curtos além da } \\
\text { possibilidade de criar seu próprio avatar. }\end{array}$ & $9+$ & Educacional & Quiz \\
\hline
\end{tabular}

Fonte: Autores.

O primeiro, "No bullying", produzido pelo Tolentinos IT Solutions, é destinado para usuários maiores de dez anos, tendo como objetivo combater o bullying nas instituições de ensino através de textos objetivos, vídeos, gamificação e um canal denúncias anônimas. Já o aplicativo australiano "Take a stand together" é destinado a usuários maiores de nove anos, e seu objetivo é fornecer informações ao usuário sobre as diferentes situações de bullying utilizando recursos audiovisuais, textos interativos e um quiz com perguntas e respostas.

O aplicativo "Take a stand together" foi desenvolvido pelo departamento de educação australiano com o objetivo de criar um ambiente na escola seguro e livre de bullying. Na tela inicial, o usuário pode criar seu próprio avatar e tem acesso a 
informativos sobre o que é bullying, onde e como acontece. Além disso, no game há possibilidade de assistir um vídeo interativo com um quiz sobre como agir em diferentes casos de bullying e cyberbullying. Importante frisar que os desenvolvedores disponibilizaram uma aba para que o usuário possa buscar ajuda se estiver passando por algumas das situações expostas durante as dinâmicas.

No aplicativo "No bullying" é necessário inicialmente fazer login, em seguida, o usuário pode se informar sobre bullying através de textos ou vídeos, contém o Quiz e tem uma aba onde é possível gerar uma denúncia de bullying anonimamente.

\section{Elaboração de um protótipo do game}

Para a elaboração de um protótipo foi escolhida a plataforma Scratch para o desenvolvimento de um game simulação que foi intitulado B.S.I.: Bullying Scene Investigation. Trata-se de um jogo de investigação onde o objetivo do participante é encontrar na escola algumas pistas de possíveis situações de bullying e cyberbullying e entregá-las na direção da instituição com um tempo hábil. Haverá três cenários para procurar as pistas: sala de aula, sala de informática e campo de futebol. A cada pista encontrada, o detetive receberá quadros informativos sobre os impactos do bullying e cyberbullying na vida das vítimas.

O cenário inicial é uma escola com alguns estudantes interagindo e o slogan do jogo B.S.I.. Para que o jogador possa avançar para o próximo cenário, basta clicar no símbolo play embaixo da tela do lado esquerdo que o jogo avança para a próxima tela. A segunda tela é a direção da escola, a diretora dá as instruções aos estudantes sobre a meta do jogo: procurar as pistas de possíveis situações de bullying e cyberbullying pelas dependências da escola. Para que a pista seja encontrada, o jogador precisa clicar com o botão esquerdo do mouse sobre a área na qual a pista foi escondida no cenário.

No próximo cenário, a sala de aula, os jogadores terão que procurar a pista em um tempo hábil, trata-se de um bilhete trocado entre alunos praticando bullying com um colega. Quando o jogador encontra a pista, um balão informativo irá conscientizá-lo sobre a incidência dos casos de bullying no Brasil. O jogador então avança para a segunda fase, a sala de informática, e ele precisa encontrar um rascunho de um e-mail com uma situação de cyberbullying. Logo após, é informado sobre o número de estudantes que sofrem de cyberbullying no Brasil.

O cenário final é durante um jogo de futebol entre os alunos, o jogador encontrará um bilhetinho que demonstra uma situação de exclusão de uma aluna nas atividades recreativas da escola, o quadro informativo que aparece logo após a pista ser encontrada alerta que essa prática também é considerada bullying (Figura 2).

A proposta de mudança de cenários após cada avanço de nível foi pensada de forma que o adolescente possa identificar as diferentes situações de bullying/cyberbullying e os impactos causados nas vítimas. Dessa forma, ao final do game, o jogador terá informações variadas sobre esse tipo de violência no ambiente escolar. 
Figura 2. Situações problema, cenários e personagens aplicados no game BSI.
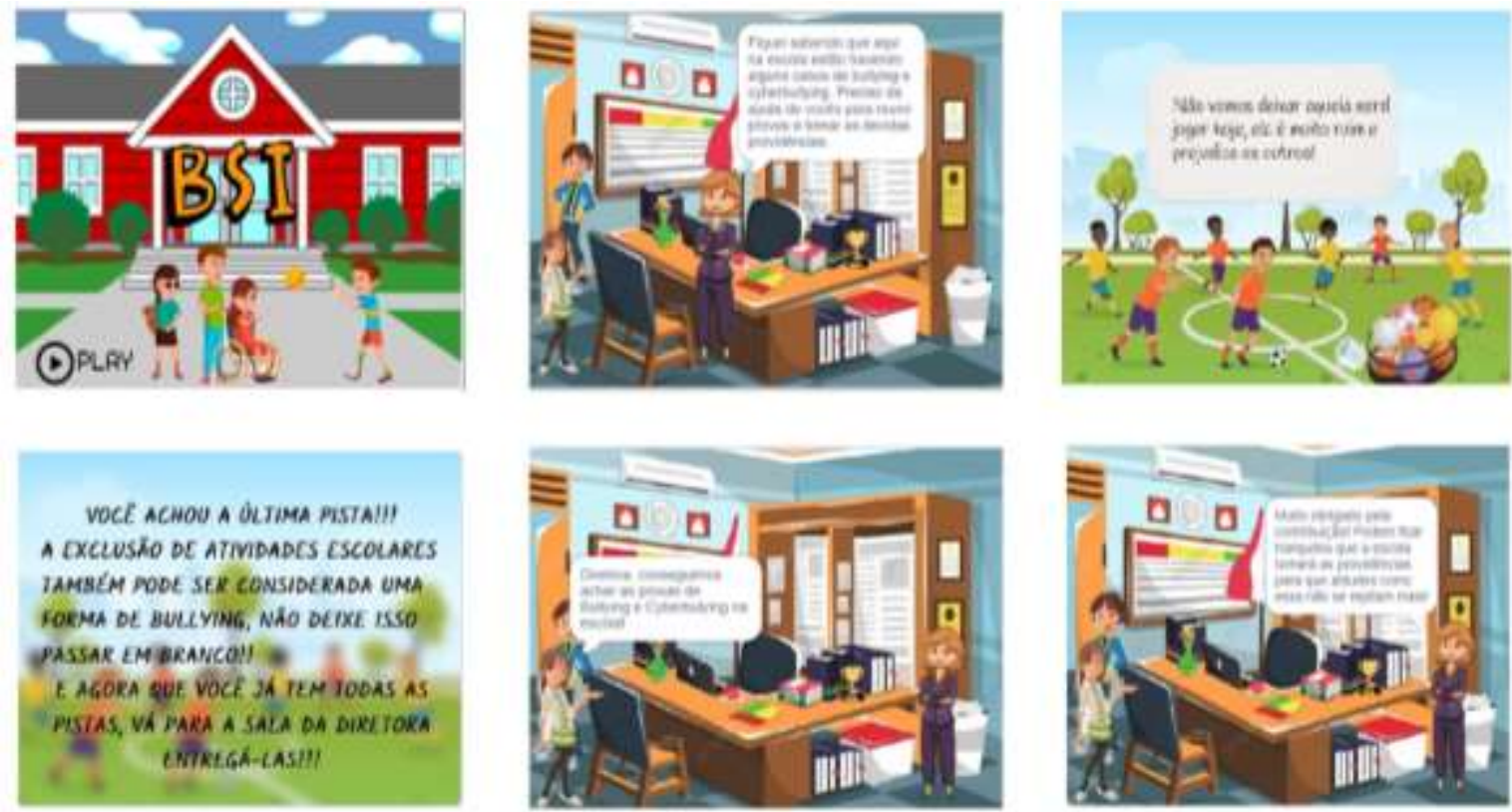

Fonte: Autores.

Após reunir todas as pistas, o jogador terá que entregá-las à diretora da escola e ela então se compromete com os estudantes a combater essas práticas de violência dentro da escola.

A utilização dos games no âmbito educacional pode trazer soluções para graves problemas educacionais, tais como a diminuição da agressividade e a eliminação da violência e dos casos de bullying e cyberbullying. Um conjunto de benefícios podem ser conquistados com a utilização da facilitação de desenvolvimento de atividades em grupo, recuperação do caráter saudável das brincadeiras e o desenvolvimento de habilidades motoras e de coordenação (Pham \& Adesman, 2015). Assim, o game pode ser um aliado no processo educativo e da formação cidadã do adolescente, pois ambientes nocivos de intensa exacerbação da violência limitam as possibilidades de aprendizado e, segundo Nomelini et al (2020), infringe o direito fundamental à educação.

Neste estudo, identificamos as características mais atraentes dos jogos de computador, estudando a literatura relacionada, e pesquisando 99 adolescentes do ensino fundamental, em uma avaliação sobre bullying e cyberbullying, o que facilitou a elaboração de uma gamificação de prevenção desses comportamentos.

Levando em consideração os efeitos deletérios desses tipos de violência, os resultados têm implicações importantes na prevenção desses comportamentos, pois fornecem evidências científicas que poderão ser eficazes na prevenção dessas ocorrências. Um elemento forte do game foi o estímulo ao protagonismo dos adolescentes em colaborar com a investigação dos casos de bullying na escola, estímulo à denúncia e, na oportunidade, pensarem em estratégias efetivas de enfrentamento. O reforço ao protagonismo de estudantes, como forma de ampliar a sua participação em espaços formativos que valorizem os talentos e habilidades pessoais na prevenção ao bullying, tem sido apontado pela literatura científica (Brandão-Neto et al., 2020; Silva et al., 2018).

A gamificação é uma ferramenta que vem sendo gradativamente efetivada no auxílio à promoção em saúde de seu público alvo. O impacto desses protótipos na aprendizagem dos adolescentes é significativo visto que, mais familiarizados com as novas tecnologias, esse grupo social demonstra maior engajamento e interesse em participar de ações que promovam atitudes e comportamentos colaborativos e um aprendizado autônomo (Pham \& Adesman, 2015). 


\section{Conclusão}

O recorte dos dados empíricos permitiu dimensionar a problemática do Bullying e Cyberbullying nas escolas, tornando-a visível, sendo este o primeiro passo quando se pretende conscientizar a comunidade escolar sobre o efeito prejudicial deste tipo de violência entre pares e a urgente necessidade de se ter métodos de prevenção. A revisão sistemática e a pesquisa de aplicativos mostraram que ainda são poucos os games neste formato proposto, disponibilizado de maneira livre e com enfoque investigativo. Assim, utilizando-se de todos os dados coletados como referência foi possível elaborar o protótipo de um game, com características que realçam o protagonismo do estudante no espaço escolar, o engajamento no combate à violência, a motivação para ajudar as vítimas de bullying e a colaboração com o professor e gestão escolar nas estratégias de enfrentamento apropriadas à realidade. Este protótipo necessita ainda ser validado para o uso em escolas. Portanto, recomendase que outras pesquisas verifiquem a efetividade de games educativos na redução do bullying.

Como limitações, o game não foi avaliado por um grupo de experts para confirmar e recomendar o conteúdo.

\section{Agradecimentos}

À Universidade de Pernambuco pelo fomento no desenvolvimento de projetos através de editais de iniciação cientifica pelo Programa de Fortalecimento Acadêmico. Às discentes, hoje enfermeiras, que realizaram a primeira fase dessa pesquisa, Alanna Cecília de Araújo Carvalho, Alessandra Aquino Guimarães e Mayara Albuquerque Dornelas de Souza.

\section{Referências}

Bayley, J. E., \& Brown, K. E. (2015). Translating group programmes into online formats: Establishing the acceptability of a parents' sex and relationships communication serious game Health behavior, health promotion and society. BMC Public Health, 15(1), 1-13. https://doi.org/10.1186/s12889-015-2545-0

Beserra, M. A., Carlos, D. M., Leitão, M. N. C., \& Ferriani, M. G. C. (2019). Prevalence of school violence and use of alcohol and other drugs in adolescents. Revista Latino-Americana De Enfermagem, 27, e3110. https://doi.org/10.1590/1518-8345.2124.3110.

Brandão Neto, W., Silva, C. O., Amorim, R. R. T., Aquino, J. M., Almeida Filho, A. J., Gomes, B. M. R., \& Monteiro, E. M. L. M. (2020). Formation of protagonist adolescents to prevent bullying in school contexts. Revista Brasileira de Enfermagem, 73(Suppl. 1), e20190418. https://doi.org/10.1590/00347167-2019-0418

Derex, M., \& Boyd, R. (2016). Partial connectivity increases cultural accumulation within groups. Proceedings of the National Academy of Sciences of the United States of America, 113(11), 2982-2987. https://doi.org/10.1073/pnas.1518798113

Ferreira, T. R. de S. C., \& Deslandes, S. F. (2018). Cyberbulling: conceituações, dinâmicas, personagens e implicações à saúde. Ciência \& Saúde Coletiva, 23(10), 3369-3379. https://doi.org/10.1590/1413-812320182310.13482018

Fiellin, L. E., Kyriakides, T. C., Hieftje, K. D., Tyra, M., Duncan, L. R., Dziura, J. D., \& Fiellin, D. A. (2016). The design and implementation of a randomized controlled trial of a risk reduction and human immunodeficiency virus prevention videogame intervention in minority adolescents : PlayForward: Elm City Stories. 1-9. https://doi.org/10.1177/1740774516637871

Gonçalves, F. G., Heldt, E., Peixoto, B. N., Rodrigues, G. A., Filipetto, M., \& Guimarães, L. S. P. (2016). Construct validity and reliability of Olweus Bully/Victim Questionnaire - Brazilian version. Psicologia: Reflexao e Critica, 29(1). https://doi.org/10.1186/s41155-016-0019-7

Goršič, M., Cikajlo, I., Goljar, N., \& Novak, D. (2017). A multisession evaluation of an adaptive competitive arm rehabilitation game. Journal of NeuroEngineering and Rehabilitation, 14(1), 1-15. https://doi.org/10.1186/s12984-017-0336-9

Jander, A., Crutzen, R., Mercken, L., Candel, M., \& De Vries, H. (2016). Effects of a Web-based computer-tailored game to reduce binge drinking among Dutch adolescents: A cluster randomized controlled trial. Journal of Medical Internet Research, 18(2). https://doi.org/10.2196/jmir.4708

Joubert, M., Armand, C., Morera, J., Tokayeva, L., Guillaume, A., \& Reznik, Y. (2016). Impact of a Serious Videogame Designed for Flexible Insulin Therapy on the Knowledge and Behaviors of Children with Type 1 Diabetes : The LUDIDIAB Pilot Study. 18(2). https://doi.org/10.1089/dia.2015.0227

Jozkowski, K. N., \& Ekbia, H. R. (2015). ““ Campus Craft ”’': 4(2), 95-106. https://doi.org/10.1089/g4h.2014.0056

Marchetti, D., Fraticelli, F., Polcini, F., Lato, R., Pintaudi, B., Nicolucci, A., \& Vitacolonna, E. (2015). Preventing Adolescents' Diabesity: Design, Development, and First Evaluation of “gustavo in Gnam's Planet." Games for Health Journal, 4(5), 344-351. https://doi.org/10.1089/g4h.2014.0107

Moher D, Liberati A, \& Tetzlaff J, A. D. (2015). Principais itens para relatar Revisões sistemáticas e Meta-análises: A recomendação PRISMA. Epidemiologia e Serviços de Saúde, 24(2), 335-342. https://doi.org/10.5123/s1679-49742015000200017

Moreira, A. P. de A., Sabóia, V. M., Camacho, A. C. L. F., Daher, D. V., \& Teixeira, E. (2014). Jogo educativo de administração de medicamentos: um estudo de validação. Revista Brasileira de Enfermagem, 67(4), 528-534. https://doi.org/10.1590/0034-7167.2014670405 
Nomelini, Q. S. S., Cunha, N. S. de S., Fernandes, R. M., Oliveira, R. R. de, Santos, C. C. R., \& Aguena, M. S. (2020). School bullying and the perception of Mato Grossense students. 2020, 1-21

Okitika, T. A., Barnabas, R. V., Rue, T., Weisman, J., Harris, N. A., Orenstein, W. A., \& Wasserheit, J. N. (2015). "Polio Eradication" Game May Increase Public Interest in Global Health. Games for Health Journal, 4(3), 195-201. https://doi.org/10.1089/g4h.2014.0045

Ortiz-Colón, A.-M., Jordán, J., \& AgredaI, M. (2018). Gamificación en educación: una panorámica sobre el estado de la cuestión SECCIÓN: ARTíCULOS This content is licensed under a Creative Commons attribution-type BY-NC. Revista Edu.Pesquì, 44, 17. https://doi.org/10.1590/S1678-4634201844173773

Panumaporn, J., Hongsanguansri, S., Atsariyasing, W., \& Kiatrungrit, K. (2020). Bystanders' behaviours and associated factors in cyberbullying. General Psychiatry, 33(3). https://doi.org/10.1136/gpsych-2019-100187

Pham, T., \& Adesman, A. (2015). Teen victimization: Prevalence and consequences of traditional and cyberbullying. Current Opinion in Pediatrics, 27(6), 748-756. https://doi.org/10.1097/MOP.0000000000000290

Programa Saúde na Escola: Caderno do Gestor do PSE. (2015). Programa Saúde na Escola: Caderno do Gestor do PSE. Secretaria de Atenção à Saúde Departamento de Atenção Básica, 68 p. Retrieved from http://bvsms.saude.gov.br/bvs/publicacoes/caderno_gestor_pse.pdf

Sápiras, F. S., Vecchia, R. D., \& Maltempi, M. V. (2015). Utilização do Scratch em sala de aula. Educação Matemática Pesquisa : Revista Do Programa de Estudos Pós-Graduados Em Educação Matemática, 17(5), 973-988. Retrieved from https://revistas.pucsp.br/index.php/emp/article/view/25152

Silva, A. N., Marques, E. S., Peres, M. F. T., \& Azeredo, C. M. (2019). Trends in verbal bullying, domestic violence, and involvement in fights with frearms among adolescents in Brazilian state capitals from 2009 to 2015. Cadernos de Saude Publica, 35(11), 1-16. https://doi.org/10.1590/0102-311X00195118

Silva, F., Dascanio, D., \& Valle, T. G. M. do. (2016). O Fenômeno Bullying: Diferenças Entre Meninos E Meninas. Reflexão e Ação, 24(1), 26. https://doi.org/10.17058/rea.v24i1.7014

Silva, J. L., Oliveira, W. A., Carlos, D. M., Lizzi, E. A. S., Rosário, R., \& Silva, M. A. I. (2018). Intervention in social skills and bullying. Revista Brasileira de Enfermagem, 71(3), 1085-1091. https://doi.org/10.1590/0034-7167-2017-0151

Sousa, M. de G., De Oliveira, E. M. L., Coelho, M. D. M. F., Miranda, K. C. L., Henriques, A. C. P. T., \& Cabral, R. L. (2018). Validation of educational game for adolescents about the sexuality topic / Validação de jogo educativo sobre sexualidade para adolescentes. Revista de Pesquisa: Cuidado é Fundamental Online, 10(1), 203. https://doi.org/10.9789/2175-5361.2018.v10i1.203-209

Stapinski, L. A., Reda, B., Newton, N. C., Lawler, S., Rodriguez, D., Chapman, C., \& Teesson, M. (2017). Development and evaluation of ' Pure Rush ': An online serious game for drug education. (November 2016). https://doi.org/10.1111/dar.12611

Vianna, M., Vianna, Y., Adler, I. K., Lucena, B., \& Russo, B. (2012). Design Thinking Design Thinking Inovação em negócios. Editora: Mjv livros.

Vrijhof, C. I., Bulk, B. G. Van Den, Overgaauw, S., Lelieveld, G., Engels, R. C. M. E., \& Van, M. H. (2016). The Prosocial Cyberball Game : Compensating for social exclusion and its associations with empathic concern and bullying in adolescents. Journal of Adolescence, 52, 27-36. https://doi.org/10.1016/j.adolescence.2016.07.005

Wendt, G. W., \& Lisboa, C. S. de M. (2013). Agressão entre pares no espaço virtual: definições, impactos e desafios do cyberbullying. Psicologia Clínica, 25(1), 73-87. https://doi.org/10.1590/s0103-56652013000100005 